Original Research Paper

\title{
Level of Non-Matching Primary Transplant Cases According to Repeat Transplants Cases and a New Bivariate Poisson Distribution
}

\author{
Ramalingam Shanmugam \\ Honorary Professor of International Studies, \\ School of Health Administration, Texas State University, San Marcos, TX 78666, USA
}

Email: rs25@txstate.edu

\begin{abstract}
In their practice, healthcare administrators and professionals often wonder about the non-matching level organs in transplants for the sake of future forecasting. Currently, there is no appropriate methodology to analyze the pertinent transplant data and describe the patterns. The lack of a suitable methodology in the literature originates from an incorrect impression that the primary transplant cases and the repeat transplant cases are two separate and independent Poisson probability processes. In fact, the actual data on the primary and repeat transplant cases in USA during the year 2014 indicate otherwise with a high degree of correlation between them. One wonders about the missing link and it hides in their model as this article articulates. The aims of this article are set to find an appropriate underlying model for the data and then construct an analytic methodology. In this research process, a novel and useful bivariate probability distribution is discovered and it is named here "seemingly independent bivariate Poisson distribution" for a lack of better title. Its statistical properties are derived, explained and illustrated. This new bivariate distribution helps not only to estimate the non-matching level of organs in the transplant cases but also to project the number of repeat transplant cases based on knowing the number of primary transplant cases and vice versa.
\end{abstract}

Keywords: Correlation, Regression, Conditional Mean and Variance, Risk

\section{Introduction}

\section{Motivation}

Organ transplantation is too important medical procedure to miss not learning enough details (Bentley, 2014 for details). Transplanting organs or tissues within the same person's body are auto-grafts. Transplants occurring between two different persons are allografts. In each scenario, non-matching transplants could occur. Tydén et al. (2012) and Urschel et al. (2013) for details on how the blood group incompatibility results in non-matching and failure in transplanted hearts. Estimating the level of non-matching is a precursor to improve the transplant process (Almond et al., 2010). Klymiuk et al. (2010) to learn about how the genetics play a crucial role in detecting non-matches of organs. The organs that have been successfully transplanted so far include hearts, kidneys, livers, lungs, pancreas, intestines and thymus. Some organs, like the brain, are not transplanted. Tissues like the bones, tendons (both referred to as musculoskeletal grafts), cornea, skin, heart valves, nerves and veins are transplantable. Most commonly transplanted organs are kidneys, followed by livers and then hearts (Gupta et al., 2015 for data). Failure in primary transplanted cases proceed to repeat transplant cases. Naesens (2014) for historical reasons for the failure of transplanted kidneys. Saczkowski et al. (2010) for reasons of the failure in the transplanted hearts. Cornea and musculoskeletal grafts are most commonly repeat-transplanted tissues. Shanmugam, 2015b) for their incidences.

How important is then the topic of transplants? For example, as of December 31, 2013, about 86,965 candidates wait for kidney transplant and it is rather a high number. Only about $83 \%$ of those candidates were awaiting for their first transplant and the remaining $17 \%$ 
waited for their repeat transplant. It is easy to realize the gravity of repeat transplant based on the numbers. Among the new candidates wait-listed for either a first time or a repeat kidney-alone transplant in 2009, the median waiting time to transplant was about 3.6 years and it suggests a great need to improve the system. Any improvement is not feasible until we learn and comprehend the information in the data. Patient survival is considerably better following repeat transplant, which is not surprising given that patients receiving second transplants are on the average younger (West, 2011). Shanmugam (2013a) for details about a technique how to estimate the chance for more survival time if a cancerous kidney is removed. Vera et al. (2009) for non-matching issues with respect to the liver transplants. In addition, there are ethical issues with respect to repeat transplants (Dobbels et al., 2012). Len et al. (2014) advocate and encourage the importance of screening and selecting organs for transplant. Whether it was for primary or repeat scenario, the transplants (Daniels, 2008; Magee et al., 2007; Piccoli et al., 2004) helped to extend the survival time. In this globalized economy, the patients in need of transplants do make a medical tour to another country and get less expensive organs transplanted (Budiani-Saberi and Delmonico, 2008).

The data for analyses and interpretations in this article are downloaded from the public domain http://optn.transplant.hrsa.gov. Motivated by the data on the primary and repeat transplant cases in USA (Table 1-4 in Eastern, Central, Mountain and Pacific regions respectively) in the year 2014 , this article is prepared to guide the data analysts on how to extract and interpret pertinent data evidence for the increased survival time. Notice that the primary and repeat transplant cases are highly correlated whether the data pertain to the Eastern, Central, Mountain, or Pacific region in USA, though individually primary as well as repeat transplant cases are seemingly follow two separate and independent Poisson probability distributions with different incidence rates. The Poisson distribution is a versatile model to capture and explain how the chance mechanism adapts to adverse rare outcomes (Shanmugam, 2013b for details).

After searching the literature, we realized that there is no appropriate probability model to fit and explain together both primary and repeat incidences data trend. Hence, we introduce a novel bivariate probability distribution and name it as "Seemingly Independent Bivariate Poisson (SIBP) distribution". This new bivariate distribution helps to predict the number of repeat transplant cases based on knowing the number of primary transplant cases and vice versa. In general, bivariate probability distributions play a key role in both the analyses and interpretations of medical or health data. Shanmugam (2015a; 2014a; 2014b; 2014c) for details about the vital role of bivariate probability distribution in health data analyses. Considering hacking versus vigilance as opposite but two different uncertain events, Shanmugam (2013c) recently developed and demonstrated a new bivariate probability distribution to assess the cyber insecurity level.

Table 1. Primary and repeat organ transplant cases in the Eastern US region during 2014

\begin{tabular}{|c|c|c|c|c|c|c|}
\hline States & $\mathrm{Y}$ & $\mathrm{X}$ & $\hat{X}$ & $\hat{Y}$ & Volatility of Y & Volatility of X \\
\hline Alabama & 14,319 & 1,634 & 1191.62 & 14122.46 & $4.58024 \mathrm{E}-06$ & 0.003082 \\
\hline Connecticut & 2,845 & 404 & 299.96 & 10383.10 & $1.15295 \mathrm{E}-06$ & 0.002266 \\
\hline DC & 13,324 & 1,699 & 1238.75 & 13798.19 & $4.76136 \mathrm{E}-06$ & 0.003011 \\
\hline Florida & 39,029 & 4,866 & 3534.61 & 22175.41 & $1.35859 \mathrm{E}-05$ & 0.004839 \\
\hline Georgia & 16,607 & 1,799 & 1311.24 & 14868.11 & $5.04 \mathrm{E}-06$ & 0.003244 \\
\hline Indiana & 11,844 & 1,304 & 952.40 & 13315.86 & $3.66072 \mathrm{E}-06$ & 0.002906 \\
\hline Kentucky & 7,222 & 731 & 537.01 & 11809.55 & $2.06411 \mathrm{E}-06$ & 0.002577 \\
\hline Maryland & 18,898 & 2,755 & 2004.28 & 15614.75 & 7.70381E-06 & 0.003407 \\
\hline Massachusetts & 26,879 & 2,865 & 2084.02 & 18215.74 & 8.01032E-06 & 0.003975 \\
\hline Michigan & 22,489 & 2,939 & 2137.66 & 16785.05 & $8.21651 \mathrm{E}-06$ & 0.003663 \\
\hline New Jersey & 17,378 & 2,230 & 1623.69 & 15119.38 & $6.24094 \mathrm{E}-06$ & 0.003299 \\
\hline New York & 53,672 & 6,730 & 4885.89 & 26947.54 & $1.87798 \mathrm{E}-05$ & 0.00588 \\
\hline North Carolina & 22,220 & 2,414 & 1757.07 & 16697.38 & $6.75364 \mathrm{E}-06$ & 0.003643 \\
\hline Ohio & 27,243 & 3,557 & 2585.67 & 18334.37 & $9.93852 \mathrm{E}-06$ & 0.004001 \\
\hline Pennsylvania & 61,171 & 8,271 & 6003.01 & 29391.46 & $2.30737 \mathrm{E}-05$ & 0.006413 \\
\hline Puerto Rico & 2,618 & 185 & 141.19 & 10309.12 & $5.42726 \mathrm{E}-07$ & 0.002249 \\
\hline South Carolina & 6,289 & 883 & 647.20 & 11505.49 & $2.48764 \mathrm{E}-06$ & 0.002511 \\
\hline Virginia & 14,563 & 1,552 & 1132.18 & 14201.98 & 4.35176E-06 & 0.003099 \\
\hline$\hat{\lambda}_{2}=\bar{x}$ & & 2,601 & & & & \\
\hline$\lambda_{1}=\bar{y}-\bar{x}$ & 18,433 & & & & & \\
\hline$\hat{\phi} \approx r \sqrt{\frac{\bar{y}-\bar{x}}{\bar{x}}}$ & 2.63 & & & & & \\
\hline
\end{tabular}


Ramalingam Shanmugam / Current Research in Medicine 2017, 8 (1): 1.13 DOI: 10.3844/amjsp.2017.1.13

Table 2. Primary and repeat organ transplant cases in Central US region during 2014

\begin{tabular}{|c|c|c|c|c|c|c|}
\hline States & $\mathrm{Y}$ & $\mathrm{X}$ & $\hat{X}$ & $\hat{Y}$ & Volatility of Y & Volatility of X \\
\hline Arkansas & 3,356 & 367 & 269.79 & 8269.91 & $1.02491 \mathrm{E}-06$ & 0.002361 \\
\hline Illinois & 33,477 & 4,597 & 3300.84 & 18006.63 & $1.25392 \mathrm{E}-05$ & 0.00514 \\
\hline Iowa & 4,508 & 703 & 510.56 & 8642.30 & $1.93952 \mathrm{E}-06$ & 0.002467 \\
\hline Kansas & 7,611 & 1,113 & 804.35 & 9645.35 & $3.05556 \mathrm{E}-06$ & 0.002753 \\
\hline Louisiana & 13,275 & 1,464 & 1055.86 & 11476.26 & $4.01101 \mathrm{E}-06$ & 0.003276 \\
\hline Minnesota & 21,156 & 3,751 & 2694.63 & 14023.83 & $1.02364 \mathrm{E}-05$ & 0.004003 \\
\hline Mississippi & 1,889 & 198 & 148.69 & 7795.69 & $5.64878 \mathrm{E}-07$ & 0.002225 \\
\hline Missouri & 13,628 & 1,369 & 987.79 & 11590.37 & $3.75241 \mathrm{E}-06$ & 0.003308 \\
\hline Nebraska & 6,317 & 1,075 & 777.12 & 9227.06 & $2.95212 \mathrm{E}-06$ & 0.002634 \\
\hline Oklahoma & 6,482 & 729 & 529.19 & 9280.40 & $2.01029 \mathrm{E}-06$ & 0.002649 \\
\hline Tennessee & 16,237 & 2,033 & 1463.58 & 12433.74 & $5.55986 \mathrm{E}-06$ & 0.003549 \\
\hline Texas & 61,445 & 6,516 & 4675.92 & 27047.39 & $1.77629 \mathrm{E}-05$ & 0.00772 \\
\hline Wisconsin & 17,673 & 2,560 & 1841.21 & 12897.93 & $6.99438 \mathrm{E}-06$ & 0.003681 \\
\hline$\hat{\lambda}_{2}=\bar{x}$ & & 2,037 & & & & \\
\hline$\lambda_{1}=\bar{y}-\bar{x}$ & 15,927 & & & & & \\
\hline$\hat{\phi} \approx r \sqrt{\frac{\bar{y}-\bar{x}}{\bar{x}}}$ & 2.53 & & & & & \\
\hline
\end{tabular}

Table 3. Primary and repeat organ transplant cases in Mountain US region during 2014

\begin{tabular}{lllllll}
\hline States & $\mathrm{Y}$ & $\mathrm{X}$ & $\hat{X}$ & $\hat{Y}$ & Volatility of Y & Volatility of X \\
\hline Arizona & 12,630 & 1,272 & 933.05 & 6713.96 & $3.59 \mathrm{E}-05$ & 0.025237 \\
Colorado & 10,907 & 1,247 & 914.86 & 6173.87 & $3.52 \mathrm{E}-05$ & 0.023207 \\
Nevada & 1,638 & 224 & 170.50 & 3268.42 & $6.56 \mathrm{E}-06$ & 0.012286 \\
New Mexico & 1,960 & 233 & 177.05 & 3369.35 & $6.81 \mathrm{E}-06$ & 0.012665 \\
Utah & 4,840 & 776 & 572.15 & 4272.11 & $2.2 \mathrm{E}-05$ & 0.016059 \\
$\hat{\lambda}_{2}=\bar{x}$ & & 750 & & & & \\
$\lambda_{1}=\bar{y}-\bar{x}$ & 6,395 & & & & & \\
$\hat{\phi} \approx r \sqrt{\frac{\bar{y}-\bar{x}}{\bar{x}}}$ & 2.67 & & & & & \\
\hline
\end{tabular}

Table 4. Primary and repeat organ transplant cases in Pacific US region during 2014

\begin{tabular}{|c|c|c|c|c|c|c|}
\hline States & $\mathrm{Y}$ & $\mathrm{X}$ & $\hat{X}$ & $\hat{Y}$ & Volatility of Y & Volatility of X \\
\hline California & 108,697 & 12,069 & 8900.09 & 44808.47 & 4.12879E-06 & 0.001686 \\
\hline Hawaii & 1,953 & 113 & 91.24 & 11586.91 & $4.23307 \mathrm{E}-08$ & 0.000436 \\
\hline Nevada & 1,638 & 224 & 173.03 & 11488.88 & $8.02696 \mathrm{E}-08$ & 0.000432 \\
\hline Oregon & 5,477 & 654 & 489.84 & 12683.67 & $2.2724 \mathrm{E}-07$ & 0.000477 \\
\hline Washington & 12,190 & 1,390 & 1032.10 & 14772.94 & 4.78799E-07 & 0.000556 \\
\hline$\hat{\lambda}_{2}=\bar{x}$ & & 2,890 & & & & \\
\hline$\lambda_{1}=\bar{y}-\bar{x}$ & 25,991 & & & & & \\
\hline$\hat{\phi} \approx r \sqrt{\frac{\bar{y}-\bar{x}}{\bar{x}}}$ & 2.80 & & & & & \\
\hline
\end{tabular}

In section 2, various statistical properties of our new SIBP distribution including their correlation, marginal, conditional probability structures are derived and then they are utilized to capture non-obvious intricacies between the primary and repeat transplant cases. One of the intricacies is an estimate of non-matching in the primary transplant cases. In section 3 , the analytic expressions are illustrated using the data on total primary versus repeat transplant cases in USA (Table 1-4) in the year 2014. Finally, in section 4, a few final comments and conclusions are compiled and stated.

\section{Seemingly Independent Bivariate Poisson Distribution and its Statistical Properties}

Let $\mathrm{Y}$ and $\mathrm{X}$ be two dependent (with a correlation, $-1 \leq \rho \leq 1)$ Poisson distributed random variables with incidence rates $\lambda_{1}>0$ and $\lambda_{2}>0$ respectively. Let $\phi>0$ be a bonding parameter, which can break down their dependence or glue together stochastically the two random variables. In the context of transplant cases, the bonding parameter portrays the non-matching level of the organ transplants. Then, we propose a 
novel seemingly independent bivariate Poisson (SIBP) distribution (1) below to model for predictability of the number of repeat transplant cases based on knowing the number of primary transplant cases and vice versa. That is:

$$
\begin{aligned}
& p\left(Y=y, X=x \mid \lambda_{1}, \lambda_{2}, \phi\right) \\
& =\left\{\frac{\left[\begin{array} { l } 
{ [ + \phi \frac { y ! } { ( y - x ) ! } ( \frac { 1 } { \lambda _ { 1 } } ) ^ { x } ] e ^ { - ( \lambda _ { 1 } + \lambda _ { 2 } ) } \lambda _ { 1 } ^ { y } \lambda _ { 2 } ^ { x } } \\
{ ( 1 + \phi ) y ! x ! }
\end{array} ; \text { for } \left\{\begin{array}{l}
x \leq y ; \\
x>y ;
\end{array}\right.\right.}{y=0,1,2, \ldots . ., \infty ; 0<\lambda_{1}, \lambda_{2}, \phi<\infty .}\right.
\end{aligned}
$$

It is obvious that $p\left(X=x, Y=y \mid \lambda_{1}, \lambda_{2}, \phi\right)>0$. After algebraic simplifications, we can show that $\sum_{y=0}^{\infty} \sum_{x=0}^{y} p\left(Y=y, X=x \mid \lambda_{1}, \lambda_{2}, \phi\right)=1$. Hence, the expression (1) is indeed a bona fide probability function. A specific probability:

$$
p\left(Y=0, X=0 \mid \lambda_{1}, \lambda_{2}, \phi\right)
$$

in (1) of no primary and no repeat transplant incidence portrays the likelihood of a healthy community. The odds for a healthy community to exist is displayed in Fig. 1 , where its odds are identified in $\mathrm{z}$-axis, the primary transplant incidence rate, $\lambda_{2}$ in $\mathrm{x}$-axis and the repeat incidence rate $\lambda_{1}$ in $\mathrm{y}$-axis). That is:

$\operatorname{Odd} s_{(0,0)}$

$=\frac{p\left(Y=0, X=0 \mid \lambda_{1}, \lambda_{2}, \phi\right)}{1-p\left(Y=0, X=0 \mid \lambda_{1}, \lambda_{2}, \phi\right)}$

$=\left(e^{\lambda_{1}+\lambda_{2}}-1\right)^{-1}$

The $\operatorname{Odds}_{(0,0)}$ in (2) for having a healthy community diminishes as the incidence rate $\lambda_{1}$ for the primary or the incidence rate $\lambda_{2}$ for the repeat transplant increases from a zero level. Likewise, the specific probability:

$$
p\left(Y=1, X=0 \mid \lambda_{1}, \lambda_{2}, \phi\right)
$$

of one primary but no repeat transplant portrays a scenario of an efficient community (because no need for repeat transplant). The odds for such an efficient is:

$$
\begin{aligned}
& \operatorname{Odds}_{(1,0)} \\
& =\frac{p\left(Y=1, X=0 \mid \lambda_{1}, \lambda_{2}, \phi\right)}{1-p\left(Y=1, X=0 \mid \lambda_{1}, \lambda_{2}, \phi\right)} \\
& =\lambda_{1}\left(e^{\lambda_{1}+\lambda_{2}}-\lambda_{1}\right)^{-1}
\end{aligned}
$$

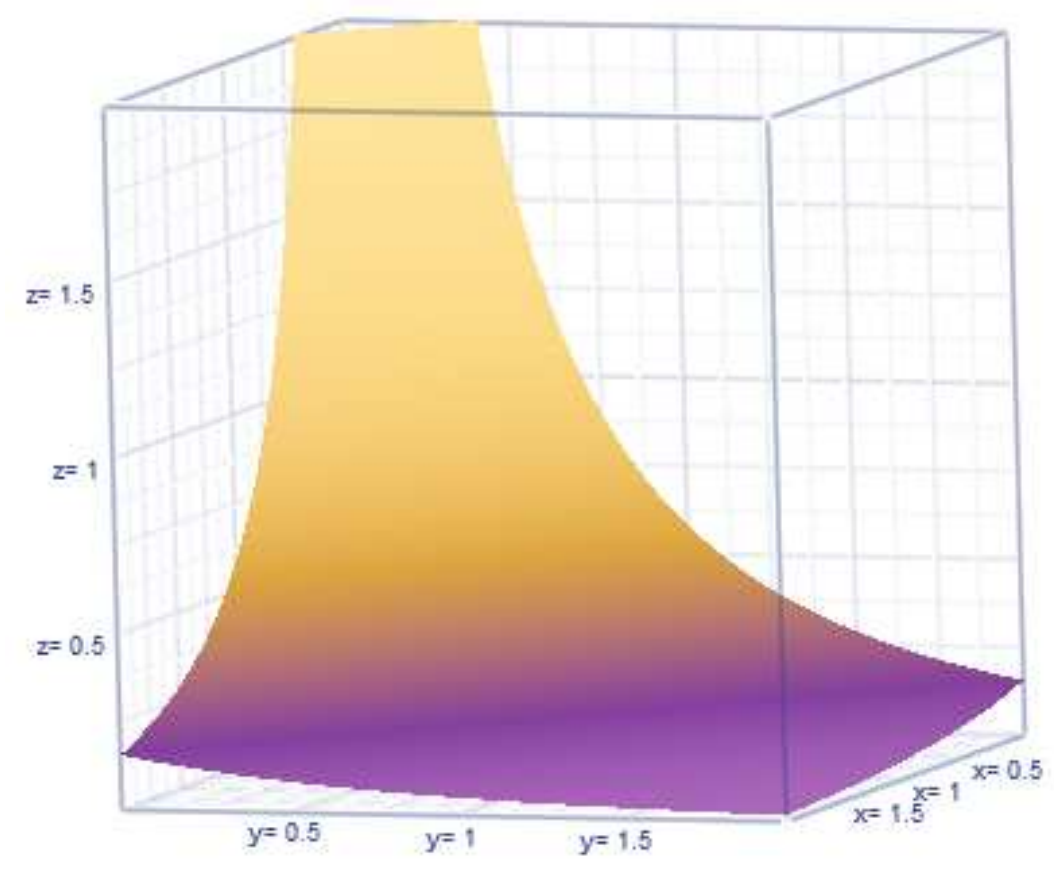

Fig. 1. Odds for healthy community 


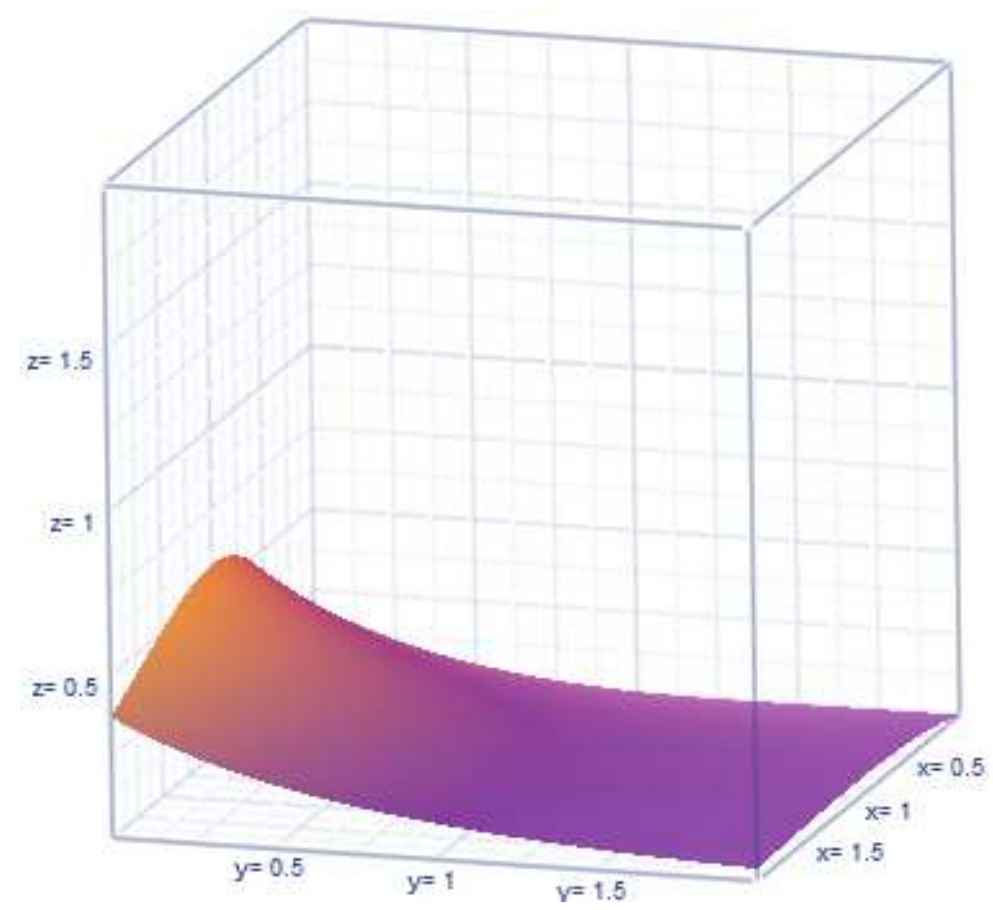

Fig. 2. Odds for efficient community

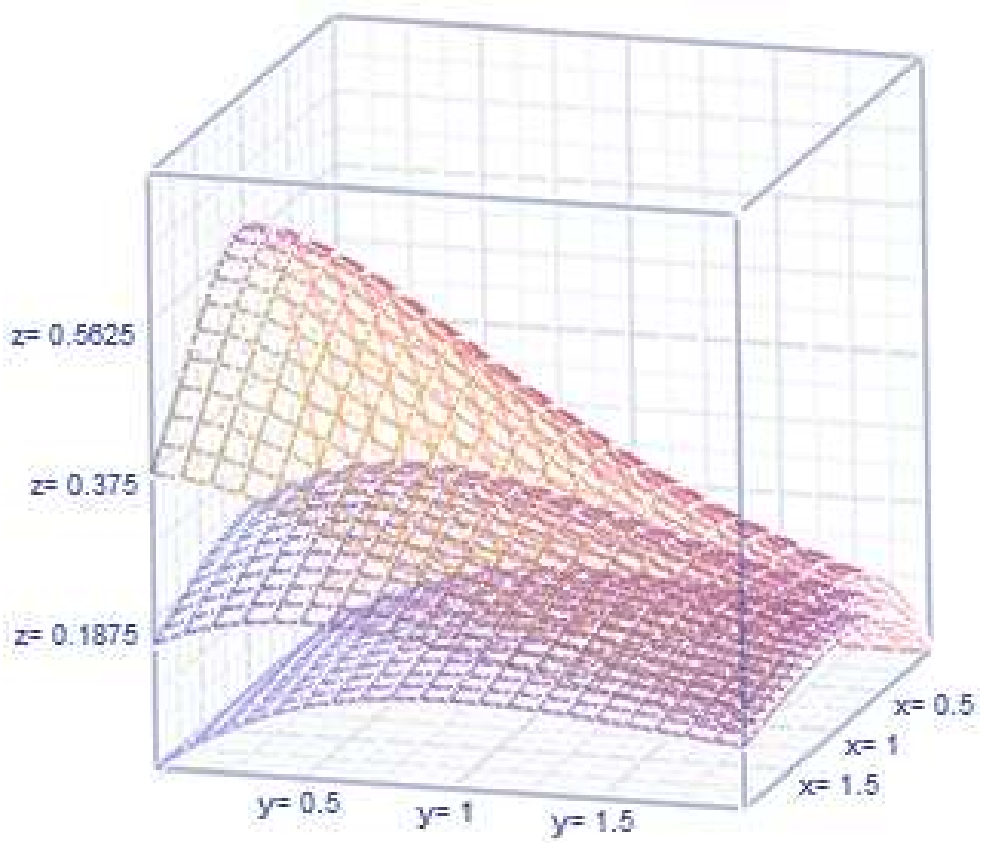

Fig. 3. Odds for inefficient community

The odds for an efficient community to exist is displayed in Fig. 2. The $\operatorname{Odds}_{(1,0)}$ starts at a finite level, depending on just the primary incidence rate $\lambda_{1}$ but concavely (Fig. 2) diminishes.

However, the probability:

$$
p\left(Y=1, X=1 \mid \lambda_{1}, \lambda_{2}, \phi\right)
$$

Portrays the likelihood of having one primary transplant and a need to have another repeat transplant (because either the primary transplant was done poorly 
or the patient's body rejects the non-matching transplanted organ or both). This scenario amounts to having an inefficient primary transplant. The odds (Fig. 3 with $\phi=0,0.5,1)$ of having inefficient primary transplant is therefore:

$$
\begin{aligned}
& \operatorname{Odds}_{(1,1)} \\
& =\frac{p\left(Y=1, X=1 \mid \lambda_{1}, \lambda_{2}, \phi\right)}{1-p\left(Y=1, X=1 \mid \lambda_{1}, \lambda_{2}, \phi\right)} \\
& =\frac{\lambda_{2}\left(\lambda_{1}+\phi\right)}{\left[e^{\lambda_{2}+\lambda_{1}}-\lambda_{2}\left(\lambda_{1}+\phi\right)\right]}
\end{aligned}
$$

where, $\phi \geq 0$ is recognized as the level of nonmatching primary transplant. In other words, with $\phi=0$, the scenario refers the absence of non-matching primary transplant. This scenario would not have a need for repeat transplant. Only in this scenario, the SIBP probability mass function (1) reduces to:

$$
\begin{aligned}
& p\left(Y=y, X=x \mid \lambda_{1}, \lambda_{2}, \phi=0\right) \\
& =\frac{e^{-\left(\lambda_{1}+\lambda_{2}\right)} \lambda_{1}^{y} \lambda_{2}^{x}}{y ! x !} \\
& =p\left(Y=y \mid \lambda_{1}\right) p\left(X=x \mid \lambda_{2}\right)
\end{aligned}
$$

It implies then that only this scenario portrays the stochastic independence between the primary and repeat transplant incidences. In a statistical sense, the bonding parameter with an attainment of a particular value $\phi=0$ breaks down the intricate relation between the primary and the repeat transplant Poisson incidences. Of course, by definition or conceptually viewing, the probability,

$$
p\left(Y=0, X=1 \mid \lambda_{1}, \lambda_{2}, \phi\right)=0
$$

of no primary but a repeat transplant is impossible. Hence, this scenario is null and void with a thought that the $\operatorname{Odd} s_{(0,1)}=0$ in a sense of degeneration.

Now, let us proceed to examine how does the number of primary transplants marginally behave without a knowledge about the number of repeat transplant cases. That is:

$$
\begin{aligned}
& p\left(Y=y \mid \lambda_{1}, \lambda_{2}, \phi\right) \\
&= \sum_{x=0}^{y} p\left(Y=y, X=x \mid \lambda_{1}, \lambda_{2}, \phi\right) \\
&= {\left[1+\phi e^{-\lambda_{2}}\left(1+\frac{\lambda_{2}}{\lambda_{1}}\right)^{y}\right] } \\
&(1+\phi) \frac{e^{-\lambda_{1}} \lambda_{1}^{y}}{y !} ; \\
& y=0,1,2, \ldots . ., \infty ; 0<\lambda_{1}, \lambda_{2}, \phi<\infty .
\end{aligned}
$$

which is nothing but a size-biased version of the regular Poisson probability mass function. The size-biased sampling has been studied by statisticians as the sampled population is quite different from the intended population in a sampling process (Shanmugam and Singh, 2012 along with the cited references there). When there is an absolute absence of non-matching transplant cases (that is, $\phi=0$ ), the number of primary transplant cases is really a separate Poisson incidences with a rate $\lambda_{1}>0$ (that is, $p\left(Y=y \mid \lambda_{1}, \lambda_{2}, \phi\right) \rightarrow \frac{e^{-\lambda_{1}} \lambda_{1}^{y}}{y !}$ as $\phi \rightarrow 0$ ). The marginal expected value and variance of the primary transplants in that scenario are then:

$$
\begin{aligned}
& E\left(Y=y \mid \lambda_{1}, \lambda_{2}, \phi\right) \\
& =\lambda_{1}+\frac{\phi}{(1+\phi)} \lambda_{2}
\end{aligned}
$$

and:

$$
\begin{aligned}
& \operatorname{Var}\left(Y=y \mid \lambda_{1}, \lambda_{2}, \phi\right) \\
& \approx(1+\phi) \lambda_{1}+\phi \lambda_{2}\left(\lambda_{2}+2\right)
\end{aligned}
$$

From the expression (7), we realize that the expected number of the primary transplants has an add-on to what it would have been when there is an absence of nonmatching transplant (that is, $\phi=0$ ). Such an add-on number is $\frac{\phi}{(1+\phi)} \lambda_{2}$ which is controlled by not only the non-matching level $\phi$ but also the repeat transplant rate $\lambda_{2}$. As mentioned in the motivation, an intricacy between the primary transplant incidences and the repeat transplant incidences is established in clear terms. The incorrect impression of seemingly unrelated occurrences of primary and repeat transplant occurrences is set correct now by the SIBP distribution (1).

Likewise, in the presence of non-matching transplant cases (that is, $\phi \neq 0$ ), the variance (7) increases from $\lambda_{1}$ (which is what it would have been in the absence of nonmatching primary transplant cases) with an add-on amount $\phi\left[\lambda_{1}+\lambda_{2}\left(\lambda_{2}+2\right)\right]$. This add-on amount suggests that the number of primary transplants is more volatile (Fig. 4) due to a higher non-matching level (that is, $\phi \neq$ 0 ) in primary transplant cases. The volatility is further controlled by a higher primary transplant occurrence rate $\lambda_{1}$ and a higher rate $\lambda_{2}$ of repeat transplant cases. This is another important intricate relation between the primary and repeat transplant cases and it would not have been possible without our new SIBP distribution (1).

Furthermore, only when $\phi=0$, the variance (7) is exactly equal to the expected value (6), validating the unique characteristics property of two separate Poisson incidences. The functional relationship between the 
variance and the mean provides unexpected clues in the data to characterize the Poisson chance mechanism (see
Shanmugam, 2014d) for details). The Fig. 5 depicts the nonlinear behavior of the variance (7).

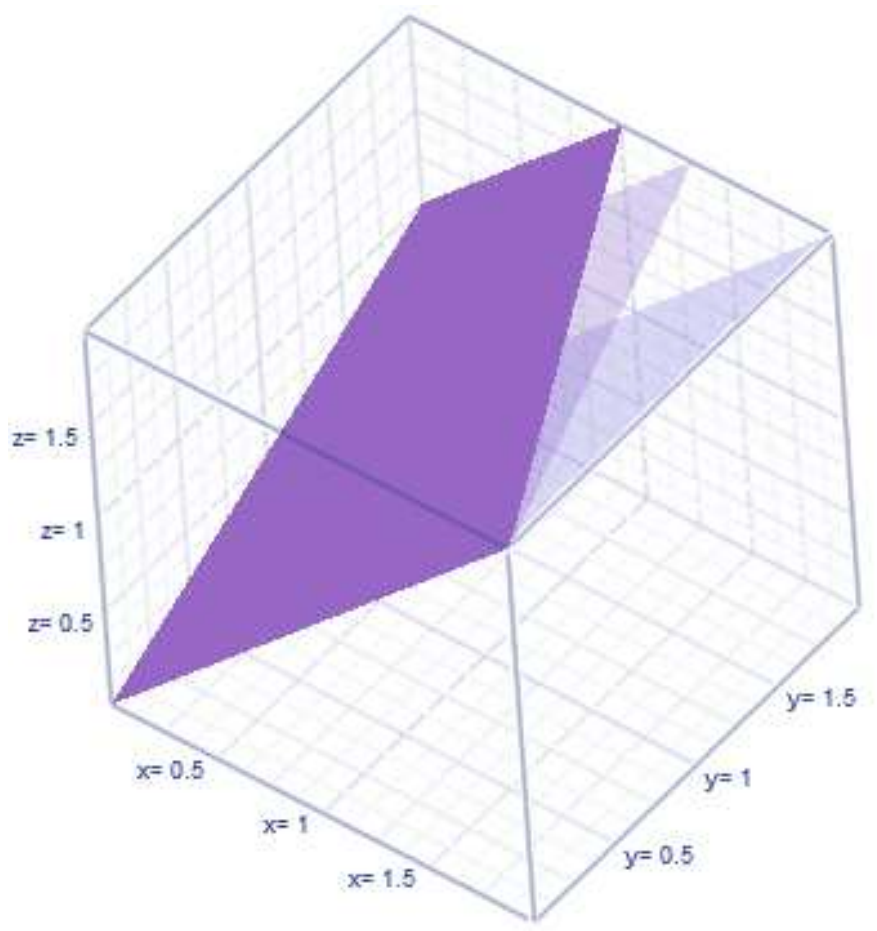

Fig. 4. Mean of SIBP with $\phi=0,0.5,1$

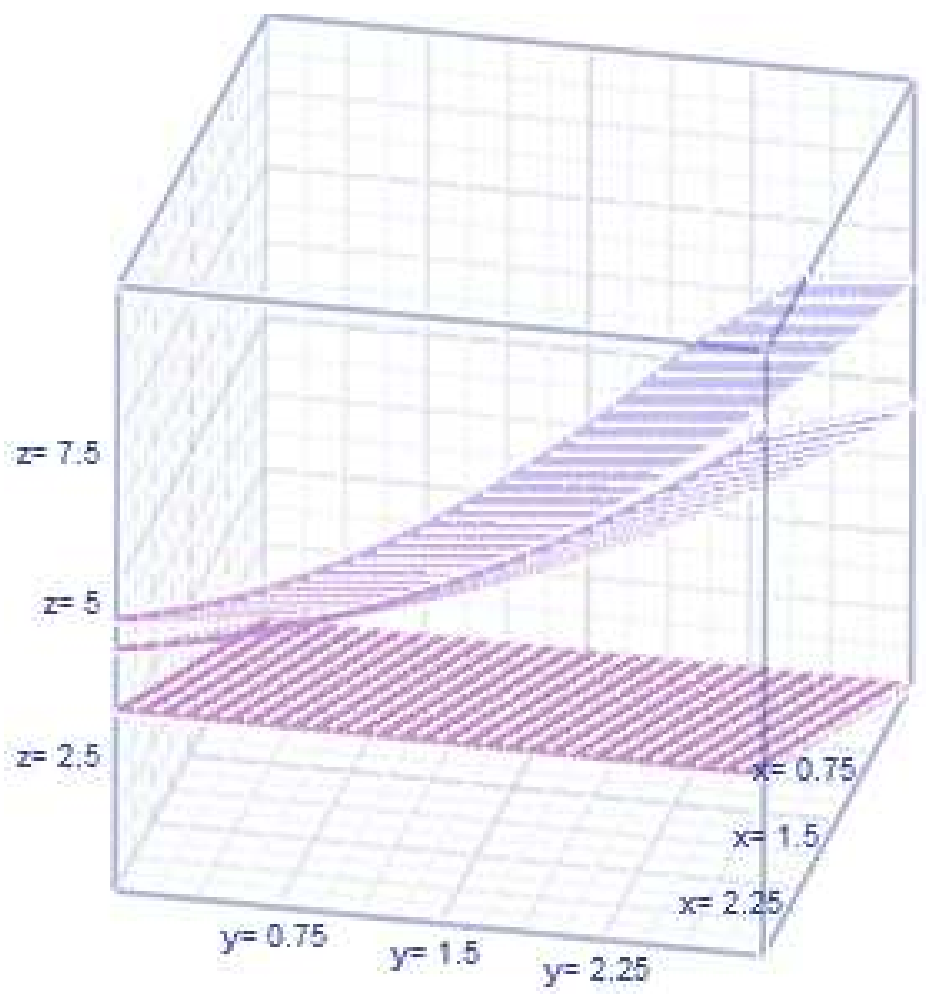

Fig. 5. Variance of SIBP with $\phi=0,0.5,1$ 
Hence, the conditional probability mass function, $p(X$ $\left.=x \mid Y=y, \lambda_{1}, \lambda_{2}, \phi\right)$ of the number of repeat transplant cases for a given marginal number $Y=y \mid \lambda_{1}, \lambda_{2}, \phi$ of primary transplants is estimable. That is:

$$
\begin{aligned}
& p\left(X=x \mid Y=y, \lambda_{1}, \lambda_{2}, \phi\right) \\
& =\frac{p\left(Y=y, X=x \mid \lambda_{1}, \lambda_{2}, \phi\right)}{p\left(Y=y \mid \lambda_{1}, \lambda_{2}, \phi\right)} \\
& =\frac{\left[1+\frac{\phi y !}{(y-x) ! \lambda_{1}^{x}}\right]}{\left[1+\phi e^{-\lambda_{2}}\left(1+\frac{\lambda_{2}}{\lambda_{1}}\right)^{y}\right]} \frac{e^{-\lambda_{2}} \lambda_{2}^{x}}{x !} ; \\
& x=0,1,2, \ldots . ., y ; 0<\lambda_{1}, \lambda_{2}, \phi<\infty .
\end{aligned}
$$

Notice that the conditional probability mass function (8) is also size-biased, but the bias vanishes in the absence of nonmatching transplant (that is, when $\phi=0$ ). In an event that there is just one primary transplant, how likely it matches so that there will be no repeat transplant? Such a probability is:

$$
\begin{aligned}
& p\left(X=0 \mid Y=1, \lambda_{1}, \lambda_{2}, \phi\right) \\
& =\frac{(1+\phi) \lambda_{1} e^{-\lambda_{2}}}{\left(1+\phi e^{-\lambda_{2}}\right) \lambda_{1}+\phi e^{-\lambda_{2}} \lambda_{2}} \\
& \rightarrow e^{-\lambda_{2}}, \text { if } \phi \rightarrow 0
\end{aligned}
$$

Furthermore, the projectile, $E\left(X=x \mid Y=y, \lambda_{1}, \lambda_{2}, \phi\right)$ of the number of repeat transplant cases for a given number $Y=y \mid \lambda_{1}, \lambda_{2}, \phi$ of primary transplant cases is:

$$
\begin{aligned}
& E\left(X=x \mid Y=y, \lambda_{1}, \lambda_{2}, \phi\right) \\
& \approx \lambda_{2}\left[1+\phi\left\{\frac{y}{\lambda_{2}+\lambda_{1}}+1\right\}\right]
\end{aligned}
$$

The projectile (9) would have been the rate $\lambda_{2}$ of the repeat transplant occurrences only in the absence of nonmatching scenario (that is, when $\phi=0$ ). The expression (9) is indeed a nonlinear regression for $X=x$ in terms of $Y=y, \lambda_{1}, \lambda_{2}, \phi$. The conditional variance (describing the volatility of the number $x$ of repeat transplants) is then:

$$
\begin{aligned}
& \operatorname{Var}\left(X=x \mid Y=y, \lambda_{1}, \lambda_{2}, \phi\right) \\
& \approx \lambda_{2}\left[1+\phi\left(\frac{\lambda_{2}}{\lambda_{2}+\lambda_{1}}\right) y(y-1)\left(1+\frac{\lambda_{2}}{\lambda_{1}}\right)^{y}\right]
\end{aligned}
$$

The conditional variance (10) is also nonlinear and it converges to the rate $\lambda_{2}$ of the repeat transplant occurrences only in the absence of no matching (that is, when $\phi=0$ ). The above intricate relations between the primary and repeat transplant cases would have been missed without the SIBP distribution (1).

Analogously, let us proceed to examine how the number of repeat transplant cases behave marginally. That is:

$$
\begin{aligned}
& p\left(X=x \mid \lambda_{1}, \lambda_{2}, \phi\right) \\
&= \sum_{y=0}^{\infty} p\left(Y=y, X=x \mid \lambda_{1}, \lambda_{2}, \phi\right) \\
&= \frac{e^{-\lambda_{2}} \lambda_{2}^{x}}{x !} ; \\
& x=0,1,2, \ldots . ., \infty ; 0<\lambda_{2} .
\end{aligned}
$$

which is, interestingly, the regular Poisson probability mass function with incidence rate $\lambda_{2}$ of the repeat transplant cases. In a marginal sense, the number of repeat transplant cases do not control the chance for non-matching of the number of primary transplant cases. There exists asymmetry in the intricate relations between the primary and repeat transplant cases. In practical terms, the asymmetry portrays traceability. Such an asymmetry would have been undiscovered without the SIBP distribution (1).

The marginal expected value and variance of repeat transplants are then:

$$
E\left(X=x \mid \lambda_{2}\right)=\lambda_{2}
$$

and:

$\operatorname{Var}\left(X=x \mid \lambda_{2}\right)=\lambda_{2}$

Hence, the conditional probability mass function, $p(Y$ $\left.=y \mid X=x, \lambda_{1}, \lambda_{2}, \phi\right)$ of the number of primary transplant cases for a given marginal number $X=x, \lambda_{1}, \lambda_{2}, \phi$ of repeat transplant cases is tractable. That is:

$$
\begin{aligned}
& p\left(Y=y \mid X=x, \lambda_{1}, \lambda_{2}, \phi\right) \\
& =\frac{p\left(Y=y, X=x \mid \lambda_{1}, \lambda_{2}, \phi\right)}{p\left(X=x \mid \lambda_{1}, \lambda_{2}, \phi\right)} \\
& =\frac{\left[1+\frac{\phi y !}{(y-x) ! \lambda_{1}^{x}}\right]}{[1+\phi]} \frac{e^{-\lambda_{1}} \lambda_{1}^{y}}{y !} ; \\
& y=x, x+1, \ldots ., \infty ; 0<\lambda_{1}, \lambda_{2}, \phi<\infty
\end{aligned}
$$

which is also a size-biased Poisson distribution with incidence rate $\lambda_{1}$. However, the size-bias, $\frac{\left[1+\frac{\phi y !}{(y-x) ! \lambda_{1}^{x}}\right]}{[1+\phi]}$ vanishes in an absence of non-matching 
transplant cases (that is, when $\phi=0$ ). In an event that there is just one repeat transplant, how likely there might had been two or more primary transplant cases. Such probability is:

$$
\begin{aligned}
& p\left(Y \geq 2 \mid X=1, \lambda_{1}, \lambda_{2}, \phi\right) \\
& =1-\frac{\left[\lambda_{1}+\phi\right] e^{-\lambda_{1}}}{[1+\phi]}
\end{aligned}
$$

Furthermore, the projectile, $E\left(Y=y \mid X=x, \lambda_{1}, \lambda_{2}, \phi\right)$ of the number of primary transplant cases for a given number $X=x \mid \lambda_{1}, \lambda_{2}, \phi$ of repeat transplant cases is:

$$
\begin{aligned}
& E\left(Y=y \mid X=x, \lambda_{1}, \lambda_{2}, \phi\right) \\
& =\lambda_{1}+\left(\frac{\phi}{1+\phi}\right) x
\end{aligned}
$$

The expression (15) is indeed a linear regression, in terms of the repeat transplant cases $X=x$, with incidence rate $\lambda_{1}$ as intercept and the proportion $\left(\frac{\phi}{1+\phi}\right)$ as its slope. The conditional variance (describing the volatility of the number $y$ of primary transplant cases) is then:

$$
\begin{aligned}
& \operatorname{Var}\left(Y=y \mid X=x, \lambda_{1}, \lambda_{2}, \phi\right) \\
& \approx\left[1+\phi x^{2}\right] \lambda_{1}
\end{aligned}
$$

The variance (16) is also nonlinear but it converges to the rate $\lambda_{1}$ of independent primary transplant occurrences, in the absence of non-matching transplant cases (that is, $\phi=0$ ). Otherwise (that is, in the presence of non-matching transplant cases indicated by $\phi \neq 0$ ), the volatility is more with an add-on amount $\phi x^{2} \lambda_{1}$ which is controlled by the non-matching level $\phi$, the occurrence rate of primary transplant cases $\lambda_{1}$ and the number $x$ of repeat transplant cases. These are extra intricate relations and revelations that would have been missed without the SIBP distribution (1).

Now, we proceed to assess the correlation between the primary and repeat transplant occurrences. For this purpose, using seemingly independent bivariate Poisson (SIBP) distribution (1), we first obtain the product moment $E(x y)$. That is:

$$
\begin{aligned}
& E(x y) \\
& =\sum_{y=0}^{\infty} \sum_{x=0}^{y} x y p\left(Y=y, X=x \mid \lambda_{1}, \lambda_{2}, \phi\right) \\
& =\lambda_{2}\left[\lambda_{1}+\frac{\phi\left(\lambda_{2}+1\right)}{(1+\phi)}\right]
\end{aligned}
$$

Substituting the expression (17) in $\operatorname{Cov}(x, y)=E(x y)$ $E(x) E y)$, we obtain that $\operatorname{Cov}(x, y)=\frac{\phi \lambda_{2}}{(1+\phi)}$. Using (7) and (13), we obtain the correlation (18) between the primary and repeat transplant cases. That is:

$$
\begin{aligned}
& \operatorname{Corr}(x, y)=\frac{\operatorname{Cov}(x, y)}{\sqrt{\operatorname{Var}(x) \operatorname{Var}(y)}}
\end{aligned}
$$

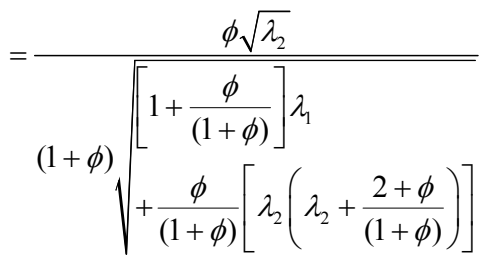

Only in the absence of non-matching transplant cases (that is, $\phi=0$ ), the primary and repeat transplant cases (namely, the random variables $x$ and $y$ ) would have been independent. Otherwise (that is, in the presence of non-matching primary transplant cases), the number of primary and repeat transplant cases are correlated, as their correlation is calculable using expression (18).

Now, we proceed to obtain estimators of the parameters: $\lambda_{1}, \lambda_{2}$ and $\phi$. For this purpose, consider a bivariate random sample $\left(x_{1}, y_{1}\right),\left(x_{2}, y_{2}\right),,,,,\left(x_{n}, y_{n}\right)$ from the seemingly independent bivariate Poisson (SIBP) distribution (1). From expression (12), notice that the estimator of the rate of repeat transplants is $\hat{\lambda}_{2}=\bar{x}$. Using expression (6), the estimator of the rate of primary transplant cases is $\hat{\lambda}_{1}=\left(\bar{y}-\frac{\phi}{(1+\phi)} \bar{x}\right) \approx \bar{y}-\bar{x}$. Let $r=\operatorname{Cor} r(x, y)$ denote the sample correlation coefficient. Then, the estimator of the non-matching level (or the bonding parameter otherwise called), $\phi$ is $\hat{\phi} \approx r \sqrt{\frac{\bar{y}-\bar{x}}{\bar{x}}}$.

\section{Illustration of Primary versus Repeat Organ Tranplant Cases in USA}

We now examine how the conceptual ideas and the derived expressions of earlier section play out in the 747,594 primary and 91,495 cases of repeat transplant cases across Eastern, Central, Mountain and Pacific regions in USA. The data for analyses and interpretations in this article are downloaded from the public domain http://optn.transplant.hrsa.gov. The primary and repeat transplant cases are categorized in the Table 1-4. The correlation between the primary and repeat transplant cases is calculated and displayed in Fig. 10 for the Eastern, Fig. 11 for the Central, in Fig. 12 for 
the Mountain and in Fig. 13 for the Pacific region of the USA. The estimate $\hat{\lambda}_{2}$ of the rate of repeat transplant cases, $\hat{\lambda}_{1}$ of the rate of primary transplant cases and $\hat{\phi}$ of the non-matching level of the primary transplant cases (also called the bonding parameter) are calculated and displayed in the tables. The projectile, $\hat{E}[y \mid x]$ of the number of primary transplant cases for a known number, $X=x$ of repeat transplant cases using (15) and the projectile, $\hat{E}[x \mid y]$ of the number of repeat transplant cases for a known number, $Y=y$ of primary transplants using (9) are calculated and displayed in the tables. Their risks:

$$
\frac{\hat{E}[y \mid x]}{\hat{V}\{\hat{E}[y \mid x]\}+\hat{E}\{\hat{V}[y \mid x]\}}
$$

and:

$$
\frac{\hat{E}[x \mid y]}{\hat{V}\{\hat{E}[x \mid y]\}+\hat{E}\{\hat{V}[x \mid y]\}}
$$

are calculated using (16) and (10) respectively and are separately displayed in the tables for the Eastern, Central, Mountain and Pacific regions in USA. In all regions, the risk for the projectiles of the repeat transplant cases is more in comparison to the risk for the projectiles of the primary transplant cases.

\section{Comments and Conclusion}

We summarize that the patterns are consistent across all (Eastern, Central, Mountain and Pacific) regions of the USA. There exists a significant discrepancy between the observed number, $Y=y$ of primary and its projectile, $\hat{E}[y \mid x]$ as much discrepancy between the observed number, $X=x$ of repeat and its projectile, $\hat{E}[x \mid y]$. There ought to be reasons for the discrepancies. Perhaps, the discrepancies are attributable not only to the inability of the model "Seemingly Independent Bivariate Poisson (SIBP) distribution" to perfectly resemble the actual reality of the transplant occurrences but also to the fact that some of the primary transplant cases might not have enough financial support to go for another round of repeat transplant, died due to other illnesses, or lost from a follow-up in the data collection process. The organ or tissue transplants are not cheap anymore. Some health insurances do reimburse their clients' air travel and/or lodging expenses to overseas hospitals in other countries if the overall medical expense for transplants are lesser expensive. However, this article provides a novel bivariate distribution and a statistical methodology to analyze and interpret the demand versus supply of organs for transplant within USA.

\section{Blue is $E(y / x)$ and Red is $E(x / y)$}

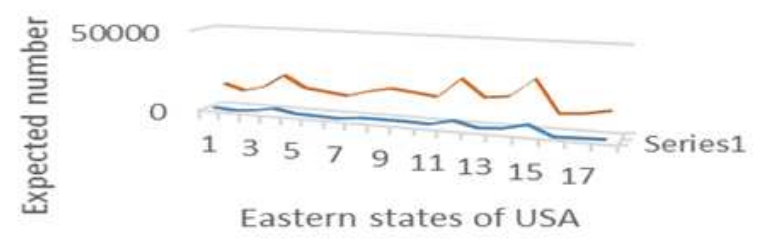

Fig. 6. Projectiles for the Eastern states of USA

$$
\begin{gathered}
\text { Blue is } E(y / x) \text { and Red is } \\
E(x / y)
\end{gathered}
$$

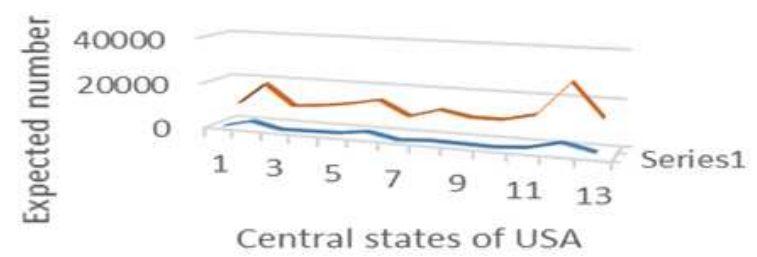

Fig. 7. Projectiles for the Central states of USA

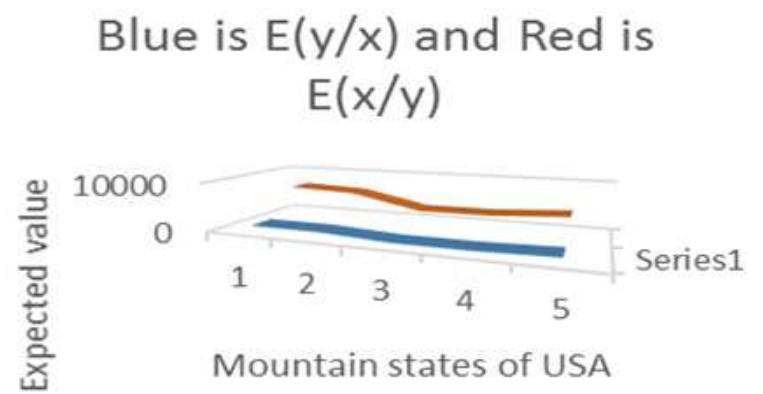

Fig. 8. Projectiles for the Mountain states of USA

$$
\begin{gathered}
\text { Blue is } E(y / x) \text { and Red is } \\
E(x / y)
\end{gathered}
$$

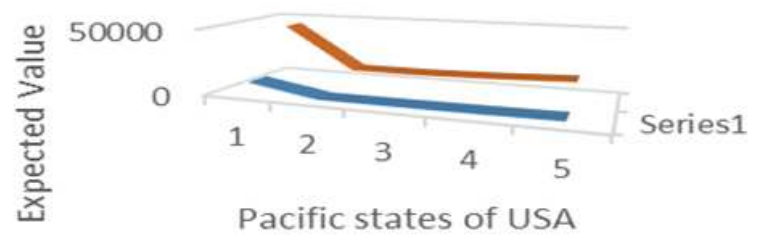

Fig. 9. Projectiles for the Pacific states of USA 
Ramalingam Shanmugam / Current Research in Medicine 2017, 8 (1): 1.13

Corr(primary, Repeat) cases $=0.99$ in Eastern USA

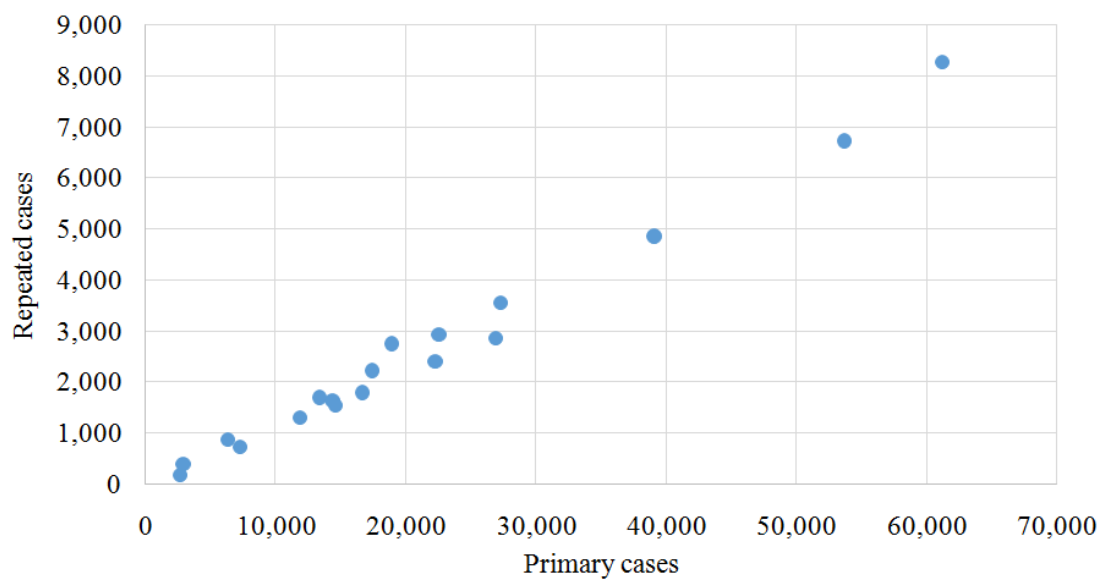

Fig. 10. For Eastern US states in 2014

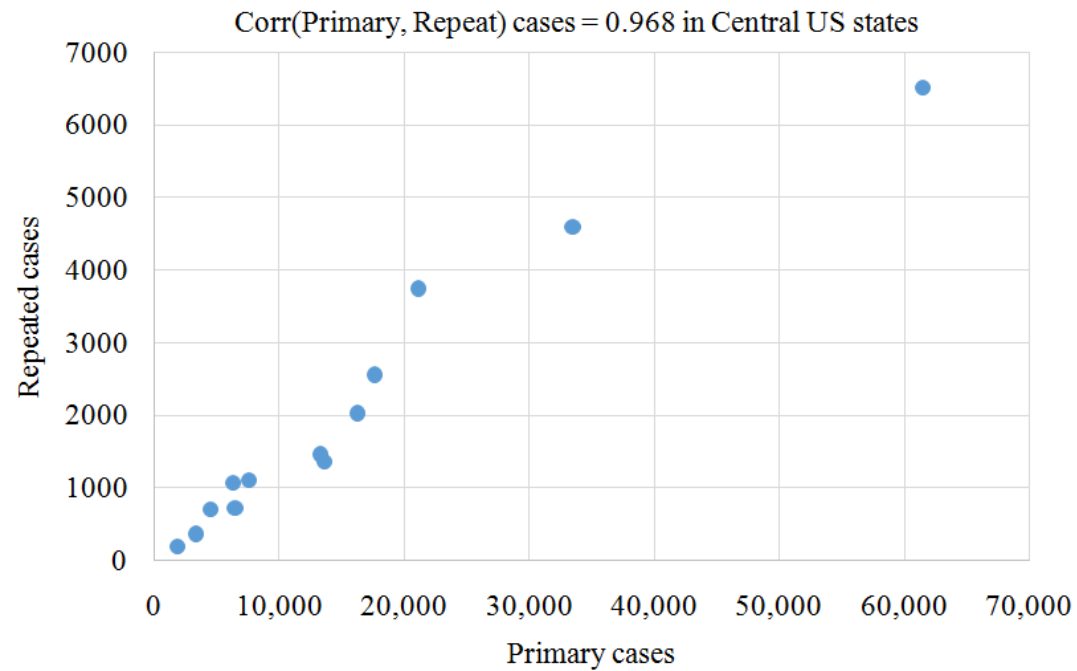

Figure 11. For Central US states in 2014

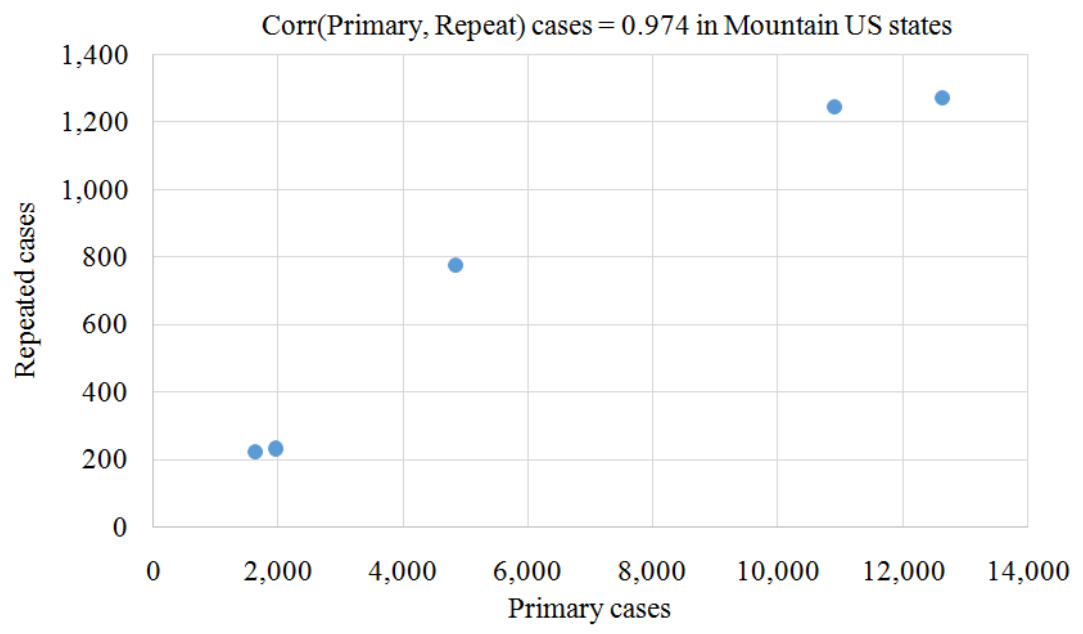

Fig. 12. For Mountain US states in 2014 


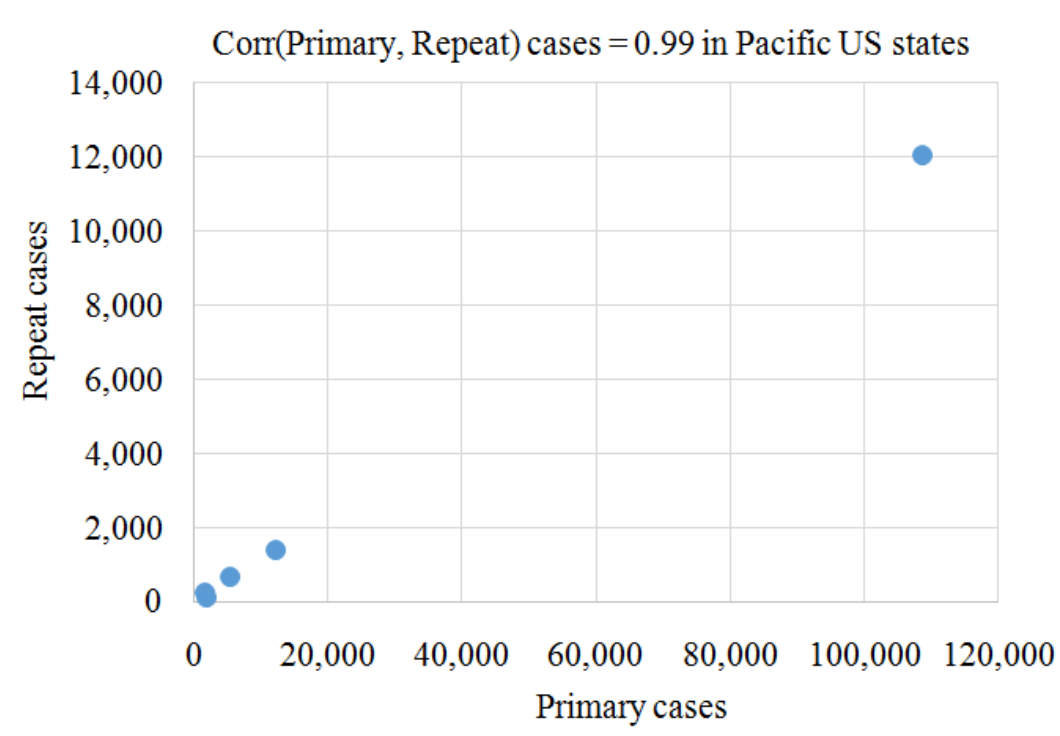

Fig. 13. For Pacific US states in 2014

A comparison (Fig. 6-9) of the conditional projectile, $\hat{E}[y \mid x]$ for the primary transplant cases versus the projectile, $\hat{E}[x \mid y]$ for the repeat transplant cases in all (that is, Eastern, Central, Mountain and Pacific) regions of the USA is made. It reveals that the conditional projectile of repeat transplant cases for given number of primary transplant cases is more consistent than the conditional projectile of the primary transplant cases for given number of repeat transplant cases. The consistency is captured using the expressions for risk. The expressions $\frac{\hat{E}[y \mid x]}{\hat{V}\{\hat{E}[y \mid x]\}+\hat{E}\{\hat{V}[y \mid x]\}}$ and $\frac{\hat{E}[x \mid y]}{\hat{V}\{\hat{E}[x \mid y]\}+\hat{E}\{\hat{V}[x \mid y]\}}$ do respectively estimate the risk of our estimate of primary transplant cases and our estimate of repeat transplant cases. Table 1-4 for the projectiles $\hat{E}[y \mid x]$ and $\hat{E}[x \mid y]$. Interestingly, the risk for projecting the number of primary transplant cases based on the number of repeat transplant cases is much smaller than the risk for projecting the number of repeat transplant cases based on the number of primary transplant cases.

\section{Acknowledgment}

The author appreciates and thanks Dr. Matthew Brooks, Director of the School of Health Administration, College of Health Professions, Texas State University for providing a release time to conduct this research work.

\section{Author's Contributions}

The contents of this article are the opinion and thoughts of the author.

\section{Ethics}

There is no violation of ethical standards in the contents.

\section{References}

Almond, C.S., K. Gauvreau, R.R. Thiagarajan, G.E. Piercey and E.D. Blume et al., 2010. Impact of ABOincompatible listing on wait-list outcomes among infants listed for heart transplantation in the United States. Circulation, 121: 1926-1933.

DOI: 10.1161/CIRCULATIONAHA.109.885756

Bentley, T.S., 2014. The U.S. Organ and tissue transplant cost estimates and discussion. Milliman Research Report, Brookfield, Wisconsin.

Budiani-Saberi, D. and F. Delmonico, 2008. Organ trafficking and transplant tourism: A commentary on the global realities. Am. J. Transplant., 8: 925-929. DOI: $10.1111 / \mathrm{j} .1600-6143.2008 .02200 . \mathrm{x}$

Daniels, N., 2008. Just Health: Meeting Health Needs Fairly. 1st Edn., Cambridge University Press, New York, ISBN-10: 052187632X, pp: 408.

Dobbels, F., A. Hames, I. Aujoulat, N. Heaton and M. Samyn, 2012. Should we transplant a patient who is non-adherent? A literature review and critical reflection. Pediatric Transplant., 16: 4-11. DOI: $10.1111 / \mathrm{j} .1399-3046.2011 .01633 . x$

Gupta, M., A. Wood, N. Mitra, S. Furth and P.L. Abt et al., 2015. Repeat kidney transplantation after failed first transplant in childhood: Past performance informs future performance. Transplantation, 99: 1700-1708. DOI: $10.1097 /$ TP.0000000000000686 
Klymiuk, N., B. Aigner, G. Brem and E. Wolf, 2010. Genetic modification of pigs as organ donors for xenotransplantation. Molecular Reproduce. Dev., 77: 209-221. DOI: $10.1002 / \mathrm{mrd} .21127$

Len, O., C. Garzoni, C. Lumbreras, I. Molina and Y. Meije et al., 2014. Recommendations for screening of donor and recipient prior to solid organ transplantation and to minimize transmission of donor-derived infections. Clin. Microbiol. Infect., 20: 10-18. DOI: 10.1111/1469-0691.12557

Magee, J.C., M.L. Barr, G.P. Basadonna, M.R. Johnson and S. Mahadevan et al., 2007. Repeat organ transplantation in the United States, 1996-2005. Am. J. Transplant., 7: 1424-31. DOI: $10.1111 /$ j.1600-6143.2007.01786.x

Naesens, M., 2014. The histology of kidney transplant failure: A long-term follow-up study. Transplantation, 98: 427-435. DOI: $10.1097 /$ TP.0000000000000183

Piccoli, G.B., G. Soragna, S. Putaggio, M. Burdese and P. Longo et al., 2004. How many organs should one patient receive? the ethics of transplantation in the medical school. Tranplant. Proc., 36: 444-445.

DOI: 10.1016/j.transproceed.2004.02.064

Saczkowski, R., C. Dacey and P.L. Bernier, 2010. Does ABO-incompatible and ABO-compatible neonatal heart transplant have equivalent survival? Interactive Cardiovascular Thoracic Surgery, 10: 1026-33. DOI: $10.1510 /$ icvts.2009.229757

Shanmugam, R., 2015a. Learning from an early start but late end epidemics via an incidence rate restricted bivariate distribution and data analysis. Int. J. Res. Med. Sci., 3: 2181-2189. DOI: $10.18203 / 2320-6012$. ijrms20150599

Shanmugam, R., 2015b. Shortage, illegal trade and unmet demand of organ or tissue transplant. J. Epidemiol. Community Med., 1: 1-9.

Shanmugam, R., 2014a. "Bivariate distribution" for infrastructures among operative, natural and no menopauses. Am. J. Biostat., 4: 34-44.

DOI: $10.3844 /$ amjbsp.2014.34.44

Shanmugam, R., 2014b. A bivariate probability model to identify "honesty" versus "cheating" in economic surveys: Xenophobia is illustrated. Am. J. Econom. Bus. Admin., 6: 42-48.

DOI: 10.3844 /ajebasp.2014.42.48
Shanmugam, R., 2014c. Probing non-adherence to prescribed medicines? A bivariate distribution with information nucleus clarifies. Am. Med. J., 5: 56-62. DOI: $10.3844 / \mathrm{amjsp} .2014 .56 .62$

Shanmugam, R., 2014d. Over/under dispersion sometimes necessitates modifying poisson model with illustration of tetanus cases and deaths after tsunami. Int. J. Ecol. Econom. Stat., 34: 37-42.

Shanmugam, R., 2013a. Tweaking exponential distribution to estimate the chance for more survival time if a cancerous kidney is removed. Int. J. Res. Nurs., 4: 29-33. DOI: 10.3844/ijrnsp.2013.29.33

Shanmugam, R., 2013b. Informatics about fear to report rapes using bumped-up Poisson model. Am. J. Biostat., 3: 17-29. DOI: 10.3844/amjbsp.2013.17.29

Shanmugam, R., 2013c. Hacking-vigilance distribution with application to assess cyber insecurity level. Int. J. Inform. Educ. Technol., 3: 300-303. DOI: 10.7763/IJIET.2013.V3.285

Shanmugam, R. and J. Singh, 2012. Urgency-biased beta distribution with application in drinking water data analysis. Int. J. Stat. Econom., 9: 56-82. 8

Tydén, G., I. Hagerman, K.H. Grinnemo, P. Svenarud and J. van der Linden et al., 2012. Intentional ABOincompatible heart transplantation: A case report of 2 adult patients. J. Heart Lung Transplant., 31: 1307-1310. DOI: 10.1016/j.healun.2012.09.011

Urschel, S., I.M. Larsen, R. Kirk, J. Flett and M. Burch et al., 2013. ABO-incompatible heart transplantation in early childhood: An international multicenter study of clinical experiences and limits. J. Heart Lung Transplant., 32: 285-292.

DOI: 10.1016/j.healun.2012.11.022.

Vera, M.E, R. Lopez-Solis, I. Dvorchik and W. Morris, 2009. Liver transplantation using donation after cardiac death donors: Long-term follow-up from a single center. Am. J. Transplant., 9: 773-881. DOI: $10.1111 / \mathrm{j} .1600-6143.2009 .02560 . \mathrm{x}$

West, L.J., 2011. ABO-incompatible hearts for infant transplantation. Curr. Opin. Organ Transplant., 16: 548-554. DOI: 10.1097/MOT.0b013e32834a97a5 